\title{
SEMIÓTICA DE LA MÚSICA: UNA TEORÍA BASADA EN PEIRCE
}

\author{
José Luiz Martínez *
}

\author{
Programa de Postgrado en Comunicación y Semiótica \\ Pontifícia Universidade Católica de São Paulo
}

El estudio de problemas de significación musical se realiza en diferentes culturas casi desde hace tanto tiempo cuanto la propia práctica de la música. Una perspectiva milenaria evocaría al menos las normas cosmo-político-musicales establecidas por los chinos; en India, el sistema musical basado en las estructuras de ragas y talas, la teoría estética de rasa (a los que se asocian ragas y talas) y la mitología y cos-

* José Luiz Martínez (São Paulo, 1960) (Ph. D. en Musicología), semioticista de la música y compositor. Sus principales áreas de investigación incluyen semiótica musical peirceana, música clásica de India, música contemporánea y danza. Martinez obtuvo el título de Doctor of Philosophy en musicología en la Universidad de Helsinki en 1997, con la tesis Semiosis in Hindustani Music, publicada por el International Semiotics Institute. En India estudió canto (dhrupad) con Ustad Zia Fariduddin Dagar. Martínez es investigador asociado al Programa de Postgrado en Comunicación y Semiótica de PUC-SP a través de FAPESP. Coordina la lista internacional de discusión de semiótica de la música Musikeion (martinez@pucsp.br). 
mología indias, marcadas esencialmente por manifestaciones sonoras; además de elementos de significación musical en las tradiciones hebrea, cristiana e islámica. No se podría ignorar la funcionalidad social y comunicativa de la música en las llamadas culturas primitivas, alrededor del globo.

Un punto de vista más próximo a la cultura occidental remitiría a las ideas relativas a música y educación social en la Grecia clásica y sus desdoblamientos medievales. Aún en la música europea, pocos períodos han sido más fértiles en especulación y práctica musical, concurriendo con la significación explícita, que el Barroco y el Romanticismo. En el primero, a través de la doctrina de los afectos, y en el segundo, con la música programática y la obra de arte total wagneriana. En realidad, la ópera ha sido desde siempre un vehículo de representaciones musicales, realizadas en diversos sistemas de acuerdo con los períodos estilísticos y las opciones estéticas de los compositores.

Sin embargo, solamente a partir de la segunda mitad del siglo XX surgieron estudios de significación musical pautados por la semiótica, en sus diversas líneas e interpretaciones. Las dificultades y deficiencias de los primeros estudios llevaron a una constante búsqueda de métodos y estrategias adecuadas a los diversos problemas relacionados con la significación en música. Tanto la semiótica de vertiente lingüística (semiología) como el estructuralismo fueron y siguen siendo explorados en este sentido. Algunas teorías han sido formuladas, siendo las más conocidas la semiología musical de Jean Jacques Nattiez $(1975,1990)$ y el enfoque greimasiano de Eero Tarasti (1994).

Además, en los últimos cinco años ha habido un crecimiento en la aplicación de la teoría general del signo desarrollada por el filósofo, lógico y científico norteamericano Charles Sanders Peirce (18391914) a análisis musicales. En especial, los escritos de David Lidov $(1986,1987)$, Robert Hatten (1994) y William Dougherty $(1993,1994)$ muestran que enfoques como éstos, correctamente organizados en ambos contextos, musicales y semióticos, tienen un poder analítico bastante grande para el entendimiento de cuestiones de significación musical. En este artículo presentaré la estructura de una teoría semiótica de la música que he desarrollado progresivamente (Martínez, 1991, 1992, 1994, 1996), y que ha sido integralmente demostrada en mi tesis doctoral, Semiosis in Hindustani Music, aprobada por la Universidad de Helsinki, en mayo de 1997 (publicada por el International Semiotics Institute). 


\section{EL SIGNO Y LA SEMIOSIS MUSICAL}

Para Peirce, los fenómenos, correspondan o no a la realidad, pueden ser comprendidos a través de las Categorías Universales: 1. primeridad, 2. segundidad, 3. terceridad (véase Peirce, 1992: 242-279 o CP 1.355368) ${ }^{1}$. En un texto de mediados de 1888 , escrito probablemente para una conferencia irónicamente denominada Trichotomic, Peirce describe de modo simple las tres categorías: «Primero es el comenzar, aquello que es nuevo, original, espontáneo, libre. Segundo es aquello que es determinado, terminado, concluido, correlativo, objeto, necesitado, reactivo. Tercero es el medio, el convertirse, desarrollar, generar» (Peirce, 1992: 280). Así, primeridad corresponde al dato cualitativo y singular del fenómeno, siendo mónada. El acaso es siempre una primeridad. En una consciencia, constituye meramente cualidades de sentimiento. La segundidad está presente en toda acción y reacción como causación efectiva. Su aspecto lógico es diádico, en una consciencia, por ejemplo, se revela cómo las ideas de ego y no-ego, o sensación (del) exterior. La terceridad es mediación, hábito o ley, no de una forma estática o rígida, sino como proceso de crecimiento. Lógicamente la terceridad se caracteriza por relaciones triádicas. En la consciencia se manifiesta como generalización, síntesis, aprendizaje o pensamiento, que ocurre siempre a través de signos. Es de suma importancia comprender que las categorías universales no son compartimentos estancos. Ellas se relacionan según el siguiente principio lógico: la primeridad es independiente; la segundidad incluye la primeridad; y la terceridad engloba la secundidad $y$, consecuentemente, la primeridad.

A partir de las categorías fenoménicas, se puede inferir que todos los fenómenos musicales (aquello que se presenta a la mente como música) ${ }^{2}$ puede constituirse en: 1 . meras cualidades acústicas (primeridad); 2. como existentes individuales (segundidad); o 3. como hábitos, leyes o signos (terceridad). Las categorías de Peirce posibilitan muchas otras clasificaciones de fenómenos musicales, cada una relativa a un determinado punto de vista. Esto ocurre, obviamente, por el hecho de que

${ }^{1}$ Las referencias a los Collected Papers of Ch. S. Peirce habitualmente son indicadas por la fórmula (CP [volumen].[párrafo]). Sus traducciones, en este artículo, son de responsabilidad del autor.

2 El concepto de «música» es, seguramente, cultural. Para una mayor riqueza en los análisis, tomaremos su sentido más amplio, donde toda y cualquier manifestación acústica, o cualquier actividad relacionada con el hacer musical, puede ser música. Se trata, evidentemente, de una concepción occidental contemporánea. 
las categorías han sido pensadas como universales: «categoría es un elemento de los fenómenos de una generalidad de primer orden» (CP 5.43). Así, es importante que se comprenda que las categorías peirceanas se manifiestan en una multitud de aspectos musicales. La propia lógica (no clásica) de la semiótica y de la clasificación de los signos de Peirce se somete a la organización triádica y a la jerarquía de los conceptos de primeridad, segundidad y terceridad. De hecho, la semiosis, «la naturaleza triádica de la operación de un signo» (CP 5.484), es un tipo de proceso en el que, genuinamente, tres correlativos toman parte:

Un signo trata de representar, al menos parcialmente, un objeto que es, por lo tanto, en un cierto sentido, la causa o el determinante de un signo, aunque el signo represente su objeto falsamente. Pero decir que él representa su objeto implica que él afecte una mente, de tal forma que, de algún modo, determina en aquella mente algo que es mediatamente debido al objeto. Esta determinación, de la cual la causa inmediata o determinante es el signo, y de la cual la causa mediada es el objeto, puede ser denominada como interpretante (CP 6.347).

De acuerdo con Peirce, el pensamiento sólo es posible mediante signos (véase CP 1.538, 4.551, 5.253). Esta noción se extiende evidentemente a la música. Un signo musical puede ser un sistema, una composición o su ejecución, una forma, un elemento interno a esa forma; o aun, un estilo, un compositor, un músico o su instrumento, y así sucesivamente. De cualquier modo, según Peirce, el proceso de significación ocurre por la relación triádica de un signo y el objeto que él representa para un interpretante que, en el caso de la música, es otro signo desarrollado en la mente del oyente, músico, compositor, analista o crítico (véase figura 1). La semiosis musical, o el proceso de significación en música, puede ser estudiada en sus relaciones entre signo, objeto e interpretante, cuando entonces la clasificación de los signos se transforma en un instrumento de análisis.

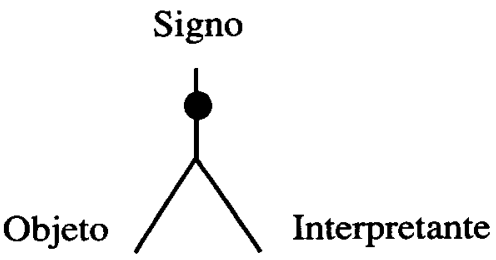

Figura 1: Diagrama triádico del signo según Peirce

(CP 3.66, 4.310). 
Cualquier cosa, de naturaleza acústica o no, puede ser un signo musical, desde que esté relacionada con algún tipo de semiosis musical. La primera serie de signos musicales se sitúa, naturalmente, en el dominio de los fenómenos acústicos. Pero hay otras posibilidades, como partituras, grabaciones, instrumentos, culturas musicales, etc. De forma general, este artículo enfocará los signos musicales acústicos; sin que, sin embargo, estén aislados de los elementos no-acústicos del universo musical.

La estructura del signo y la naturaleza de su acción, o semiosis, se desdobla en la clasificación de los signos. Peirce la ha pensado, en 1903, en tres tricotomías (CP 2.243). Se trata de las relaciones básicas entre los tres correlativos de la semiosis: el signo en relación consigo mismo, el signo en relación con su objeto, y el signo en relación con su interpretante. Cada tricotomía da origen a tres tipos de signos (CP 2.244-252) y su combinación lógica proporciona diez clases de signos (CP 2.254-263). En la Tabla 1, se puede verificar cómo las tricotomías básicas están relacionadas con los conceptos de signo, objeto e interpretante, estando sometidas a la arquitectura lógica de la primeridad, segundidad y terceridad:

\section{Tabla 1 - Tricotomías peirceanas}

\begin{tabular}{lccc}
\hline & El signo & El signo & El signo \\
& en sí mismo & y su objeto & $y$ su interpretante \\
\hline Primeridad & Cualisigno & Icono & Rema \\
Secundidad & Sinsigno & Índice & Dicente \\
Terceridad & Legisigno & Símbolo & Argumento \\
\hline
\end{tabular}

Se podría probar una ejemplificación musical simple de esos nueve tipos de signos. Para la primera tricotomía, el signo en relación consigo mismo: cualisigno, una cualidad, por ej., un timbre; sinsigno, un existente individual, por ej., una ejecución de la Sinfonía Fantástica de Berlioz; legisigno, una ley o convención, por ej., la forma sonata. Para la segunda tricotomía, el signo en relación con su objeto: icono, un signo que representa su objeto por el hecho de que, en sí mismo, posee cualidades semejantes a él, por ej., los cantos de pájaros en la Escena junto al arroyo de la Sinfonía Pastoral de Beethoven; índice, un signo que representa su objeto por el hecho de que se relaciona dinámicamente con él, por ej., un solo de sitar como signo de la música de India; símbolo, un signo que será interpretado como refiriéndose a su objeto meramente debido a un hábito o convención, por ej., un himno 
nacional, un leitmotiv. Para la tercera tricotomía, el signo en relación con su interpretante: rema, un signo que para su interpretante constituye apenas una posibilidad, por ej., la audición de un concierto de música aleatoria; dicente, un signo que para su interpretante es un signo de facto, por ej., el reconocimiento de una determinada composición en un concierto; argumento, un signo que para su interpretante es un signo de ley, por ej., un análisis musical en una revista especializada.

\section{TAMBORES DE PAPUA-NUEVA GUINEA, UN EJEMPLO}

Sin embargo, la multiplicidad de la semiosis musical no puede ser evaluada brevemente. Signo, objeto e interpretante se imbrican en relaciones e interrelaciones. Considere el Ejemplo 1. En él, el aspecto material del signo ya está bastante formalizado por la notación musical. Su ejecución, en un instrumento cualquiera, produce un cierto aspecto acústico. En realidad, ésta sería apenas una de las diversas posibilidades de interpretación que la notación musical ofrece. Tanto la configuración gráfica como la acústica son signos. Hay una amplia gama de posibles interpretantes para esos signos que, por su lado, se refieren diferentemente a sus posibles objetos. Es necesario estudiar caso a caso, de acuerdo con los diversos puntos de vista.

\section{Ejemplo 1}

(Papua-Nueva Guinea, de la cultura Nor-Papua. Schmidt in Schneider, 1957: 82)

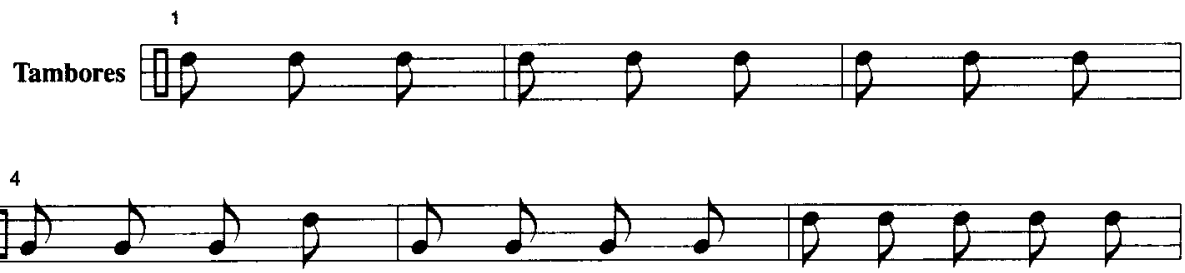

Lo que se ofrece primero a los oídos es la realización acústica. En el Ejemplo 1, en su tonalidad (es decir, sin ninguna otra referencia a cualquier objeto, considerándose apenas sus propias cualidades timbrísticas y rítmicas), aquello que aflora es su materialidad musical. Para un oyente que desconozca, o que sea capaz de abstraer el origen y la organización musical del Ejemplo 1, es decir, concentrarse en la 
materialidad, el signo será un cualisigno. En relación con su objeto, en este caso, sus propias cualidades acústicas, es un icono casi puro. Un signo que se refiere a sí mismo. En esas condiciones, su interpretante será un rema, es decir, un signo de posibilidad; probablemente presentándose como cualidades de sentimiento, en la mente del oyente.

Para un etnomusicólogo, o para un aficionado a world music, o para quien quiera que comprenda que el Ejemplo 1 es una manifestación de la cultura de los Nor-Papua de Papua-Nueva Guinea, el signo será un índice en relación con su objeto, en esa cultura en particular. Ambos constituyen existentes de hecho, la ejecución del Ejemplo 1 (signo) se relaciona con los Nor-Papua (objeto) de manera factual. Una cultura y sus determinaciones históricas, geográficas, antropológicas, etc., determinando un signo musical, el índice genuino es el resultado de una relación de causa y efecto, o mejor, de acción y reacción; sin embargo, esa relación no es una segundidad pura, porque ella está vinculada a un tercero, un significado, el interpretante del índice. La notación musical resulta de investigación en campo, posiblemente de una grabación in loco ${ }^{3}$. Su interpretante será un dicente, constatando la relación dinámica entre signo y objeto.

Ahora, tómese un individuo de aquella cultura, familiarizado con el significado convencional del signo, como invitación para fumar tabaco (de acuerdo con Schmidt en Schneider, 1957: 82). Aquí, el signo se refiere a su objeto (la invitación) no en relación con su materialidad acústica, ni por el hecho de ser un índice de los Nor-Papua, pero debido a una relación establecida por hábito. Desde este punto de vista, es un legisigno. En relación con su objeto, un símbolo. Su interpretante de facto (dinámico) podría ser del tipo emocional (una sensación de euforia, por ejemplo), energético (la actitud de dirigirse a la aldea que ha emitido el símbolo) o lógico (un silogismo como si hay invitación, habrá un ritual o una fiesta). En este último caso, el signo interpretante sería un argumento.

Se debe notar que cada situación semiótica destacada anteriormente es una complejidad dependiente de muchos factores y puntos de vista. Las diversas modalidades de semiosis, en un caso concreto, no están

${ }^{3}$ En rigor, como entidad original registrada gráficamente o congelada por la grabación, constituye un existente singular, un sinsigno. Su registro como partitura o grabación (sea mecánica, magnética o digital) depende de codificaciones formales que pueden ser clasificadas como legisignos. Por presentar similitudes con la música original, se trata, por lo tanto, de un legisigno icónico. 
aisladas unas de las otras. Hay varias líneas y planos de interdependencia entre los modos de significación. Se puede decir que el símbolo incluye el índice, y que el índice incluye el icono. En un acontecimiento efectivo del Ejemplo 1, un individuo Nor-Papua podría percibir simultáneamente el timbre particularmente brillante de los nuevos instrumentos (icono), además del hecho de que los sonidos vienen de la región norte (índice), además de la significación convencional de la invitación para fumar (símbolo). Un semioticista podría destacar éstos u otros planos para su estudio. En realidad, el análisis semiótico podría ser pensado como el recorrer un laberinto multidimensional.

\section{CAMPOS DE INVESTIGACIÓN SEMIÓTICA}

La teoría semiótica de la música que propongo abarca tres campos interrelacionados de investigación: 1. Semiosis Musical Intrínseca, o el estudio del signo musical consigo mismo. En este campo se trabaja con la significación musical interna. Se trata de la semiótica de la materialidad musical. La semiosis intrínseca abarca el problema de las cualidades musicales, la realización de obras y estructuras musicales, y los hábitos de organización en la música, tales como sistemas musicales, estilos y escuelas. 2. Referencia Musical, o el estudio de los signos musicales relacionados con sus posibles objetos. Se trata aquí de la investigación de las representaciones musicales de una variedad de objetos acústicos o no acústicos. Las principales cuestiones de este campo incluyen: cómo un signo musical se refiere a su objeto, los posibles objetos representados por la música y sus modos de existencia, las posibles relaciones entre un objeto y la manera como lo representa el signo. 3. Interpretación Musical, o el estudio de los signos musicales relacionados con sus interpretantes. En este campo se investiga la acción de los signos musicales en una mente potencial o existente. Las cuestiones de interpretación musical pueden ser divididas en tres subcampos: (3.1) percepción musical; (3.2) ejecución; y (3.3), subdividido en inteligencia musical y composición. La inteligencia musical abarca análisis, crítica, educación, teoría de la música y semiótica musicales. En mi tesis doctoral (Martínez, 1997), esta teoría ha sido aplicada a una variedad de aspectos de la música clásica del norte de India, en especial a la teoría estética que fundamenta los ragas, la teoría de la esencia estética o rasa en sánscrito. Así, la músi- 
ca indostaní ha sido el primer objeto de estudio de la teoría semiótica de la música que he propuesto. Sin embargo, creo que esta teoría puede ser usada como modelo para analizar otras tradiciones musicales.

\subsection{Semiosis musical intrínseca}

Vamos a considerar, dentro de esta estructura teórica, posibles capas de significación musical. En el campo de la semiosis musical intrínseca, antes de nada, cada obra o su realización presenta cualidades musicales peculiares. Se trata aquí no sólo de las cualidades timbrísticas, rítmicas y melódicas, sino también de la cualidad musical general que un cierto signo puede tener. Las diferentes maneras de utilizar la voz humana en las diversas músicas del planeta son una referencia inequívoca de la variedad de cualidades musicales posibles. De hecho, la voz produce cualidades únicas, explícitas si pensamos en los juegos vocales Inuit, en el bel canto, en el khayal del norte de India, en el teatro no japonés, o en las canciones de los trovadores africanos griot; cualidades que constituyen ejemplos de cualisignos musicales. Por otro lado, la música significa en la relación de sus formas y estructuras con la realización de éstas en la composición, en la ejecución o en la improvisación. Formas y estructuras, hábitos musicales de organización, son comprendidas semióticamente como legisignos; mientras que sus realizaciones, ejecuciones de formas y de obras, constituyen sinsignos, existentes musicales. De esta forma, la cadencia tonal clásica, por ejemplo, se manifiesta efectivamente en todas las músicas que hacen uso de ella.

\subsection{Referencia musical}

El campo de la referencia musical, estudiando las relaciones entre signo y objeto, revela la capacidad de la música en representar una gran variedad de objetos acústicos y no acústicos. La música significa en varios niveles y el más fundamental es aquel en que signo y objeto presentan una relación de identidad, se trata de la idea de icono puro. La música posee una capacidad de significación autónoma tan grande que algunos estetas, como Eduard Hanslick (1989: 61), y compositores, como Pierre Boulez 
(1986: 32) y John Cage (1961: 96; Kostelanetz, 1988: 200), propusieron que éste es su principal modo de operar. Sin embargo, la idea de la música pura o absoluta no puede ser extendida a todas las otras músicas, en donde representaciones de diversos tipos sostienen concepciones estéticas distintas. Consideremos el tercer movimiento de la Sinfonía de Luciano Berio. Tenemos aquí el procedimiento composicional de la citación. Berio teje este movimiento de la Sinfonía con fragmentos citados de diversos compositores. En los diez primeros compases, los trombones y las tubas, y después las cuerdas, ejecutan un fragmento de la cuarta parte de los Fünf Orchesterstücke de Schoenberg. Sobrepuestos, trechos de la Cuarta Sinfonía de Mahler (en las flautas, percusión y cuerdas) con Jeux de Vagues (maderas, arpa, timbales y cuerdas), la segunda parte de La Mer de Debussy. La Segunda Sinfonía de Mahler es citada luego, enseguida, en las maderas (véase Hicks, 1981). Todos esos fragmentos son signos icónicos, que yuxtapuestos y entretejidos con hablas del coro, en especial de la obra de Samuel Beckett The Unnamable, engendran una fabulosa metáfora musical (véase Martínez,1996). Por otro lado, la Sinfonía es un existente en tiempo y espacio, y así es un índice de la música tal como es concebida por Berio. Toda ejecución de esta obra es un signo indicial de la música europea en la segunda mitad de este siglo; $o$, más particularmente, de la música concebida por un cierto individuo. Finalmente, la Sinfonía de Berio puede ser pensada como un símbolo. Con el desarrollo del tercer movimiento, Berio incluye citaciones de un número aún mayor de compositores: Berg, Brahms, Hindemith, Ravel, Strauss, Berlioz, Stravinsky, el propio Berio, Pousser, Beethoven, Boulez, Webern y Stockhousen, siempre entretejidas con Mahler. Con el coro presentando la frase de Beckett: Where now? Who now? When now? la Sinfonía de Luciano Berio, en su totalidad, constituye un símbolo preñado de significados, no solamente de la propia historia de la concepción sinfónica de música, sino también de la complejidad del universo musical contemporáneo; en donde los signos musicales, de todas las épocas, se desdoblan continuamente y yuxtaponen sus universos de significado, generando nuevos signos y nuevos significados.

\subsection{Interpretación musical}

Toda la música es un complejo semiótico que puede ser estudiado a partir de diversos puntos de vista. Los campos de investigación musi- 
cal favorecen una especificación precisa del análisis semiótico. Sin embargo, es necesario considerar que la lógica que rige la semiótica de Peirce establece la interdependencia de los tres campos de investigación. La semiosis musical intrínseca, aunque pueda ser estudiada de forma independiente, está inserta en el campo de la referencia musical. Estos dos campos, por su lado, se insertan en la interpretación musical (véase figura 2). De esta forma, la interpretación musical constituye el dominio más amplio y complejo de la semiosis musical. Los interpretantes musicales, como proceso semiótico, son el resultado efectivo de la significación musical. Aunque la interpretación musical dependa de la semiosis intrínseca y de la referencia musicales, es en la complejidad del campo de los interpretantes donde la música realmente se presenta, existe y significa.

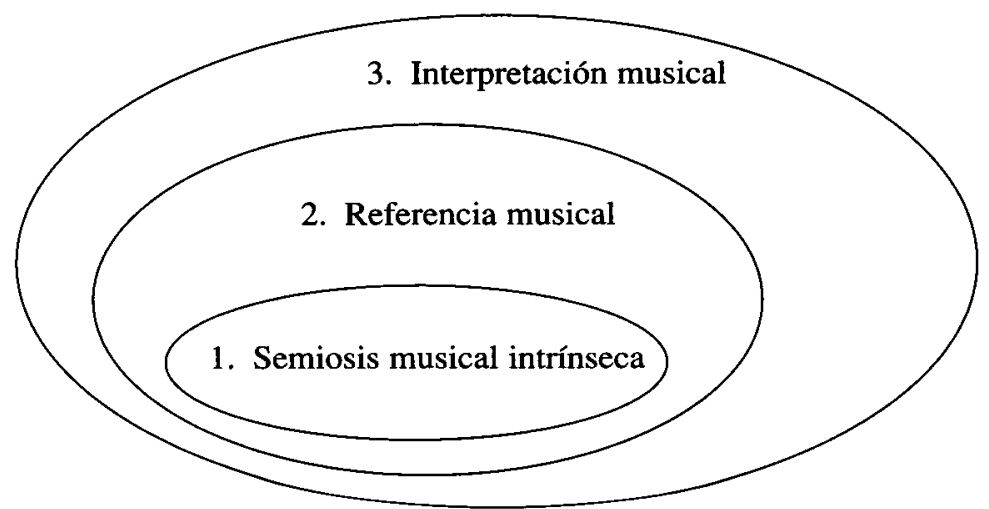

Figura 2: Relaciones lógicas entre los tres campos de investigación semiótica.

El campo de la interpretación musical constituye un grupo de disciplinas interrelacionadas (véase Tabla 2). La percepción (y la cognición), la ejecución, la inteligencia y la composición son formas de interpretantes musicales porque son el resultado de la semiosis de facto. La percepción es -obviamente- el punto inicial del proceso efectivo de generación de interpretantes musicales. En primer lugar, la percepción y la cognición son seguramente esenciales en el proceso de formación de una mente musical. En segundo lugar, la semiosis musical se origina en la percepción, pues es a través de la participación de una mente por lo que la música es disfrutada, realizada, concebida, transformada, ejecutada, enseñada y compuesta. He dividido el sub- 
campo de la percepción musical en tres áreas de estudio, de acuerdo con los interpretantes emocionales, energéticos y lógicos. Subdivisiones aún más finas se presentan en la Tabla 2.

La ejecución ${ }^{4}$ es una forma de interpretante musical al mismo tiempo necesario y complementar a la percepción, pues trata de la efectivación de signos musicales. Hay un componente de fuerza bruta - segundidad, o causación eficiente- en la ejecución, el impacto físico que causa la vibración sonora de los instrumentos. Pero la ejecución no es meramente pura fuerza bruta, pues existen - casi siempreprincipios inteligentes controlando los impulsos psicomotores o los artefactos electroacústicos que produce la música. Se trata de formas de causación final - terceridad- que rigen los procesos de causación eficiente (véase CP 1.211-212). El subcampo de la ejecución musical puede ser dividido en: realización orientada en aspectos musicales, realización orientada en aspectos funcionales y realización orientada en aspectos representativos.

El tercer subcampo de la interpretación musical está compuesto por las áreas de la inteligencia musical y de la composición. La inteligencia musical consiste en las formas científicas de pensar la música. Diría que la musicología, en su sentido más amplio, está inserta en este subcampo. En su epistemología, es necesario considerar que el universo musical, como también el universo físico y mental, está en estado continuo de expansión. Así, la verdad que puede ser establecida por las ciencias de la inteligencia musical constituye verdades transitorias, pues el proceso de investigación musical es abierto, continuo e infinito. Cualquier investigación musical es necesariamente semiótica, pues el único modo de conocer la realidad es a través de los signos y de la semiosis. Del modo como ha sido entendido por Peirce, las formas lógicamente más elevadas de semiosis, el pensamiento racional y simbólico, se caracterizan por la continuidad: la traducción de un signo a otro, mejor desarrollado. Este proceso no es nada más que el desdoblar de los interpretantes. He subdividido este subcampo en tres áreas: estética musical, o el estudio de lo que es admirable en música; pragmática musical, el estudio de los aspectos prácticos de la música; y semiótica musical, la disciplina que estamos ejerciendo en este momento.

4 Se puede incluir aquí la dirección (de orquesta, coral) como una forma de metaejecución musical. 
Finalmente, la composición ${ }^{5}$ constituye un sub-campo paralelo a la inteligencia musical. Una clasificación semiótica de las composiciones puede ser establecida en tres tipos: música absoluta, en donde el signo musical no hace ninguna referencia a un objeto que no sea su propia materialidad musical; música funcional, en donde existe una relación dinámica de la música con alguna otra cosa o estructura semiótica; y música representativa, en la que signos musicales a través de procedimientos icónicos, indiciales y/o simbólicos hacen referencia a fenómenos acústicos y no-acústicos.

La estructura aquí propuesta de una teoría semiótica de la música, abarca - como es evidente - todo el ámbito del pensamiento musical, proponiendo una clasificación que, lejos de ser rígida y dogmática, pretende ser flexible y en constante actualización. En la apreciación de esta teoría es importante recordar que, como un laberinto multidimensional, los diversos campos y subcampos se sobreponen e interactúan de acuerdo con la lógica de los relativos, que puede ser sintetizada por la fór-

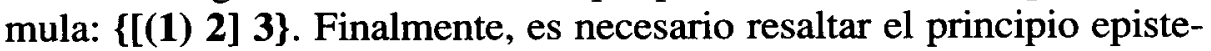
mológico que comparto; a saber, esta teoría, por más completa que sea, asumirá siempre configuraciones particulares en cada aplicación que se haga de sus principios. De hecho, la ciencia, como signo de la realidad, no determina el objeto, sino que el objeto - en este caso el hecho musical complejo- es quien determina el signo y sus interpretantes.

\section{Tabla 2 - Interpretación musical}

\subsection{Percepción (y cognición) musicales}

3.1.1. Audición emocional

3.1.1.1. Cualidad de sentimiento

3.1.1.2. Sensación

3.1.1.3. Emoción

3.1.2. Audición energética

3.1.2.1. Esfuerzo mental

3.1.2.2. Movimiento corporal instintivo

3.1.2.3. Aplauso, trabajo, danza

3.1.3. Audición racional

3.1.3.1. Abducción musical

3.1.3.2. Inducción musical

3.1.3.3. Deducción musical

5 Se puede incluir aquí tanto el arreglo y orquestación como la improvisación (basada en un sistema específico, como una de las corrientes de jazz o la música indostaní, o improvisación libre, en la medida que cualquier improvisación puede ser realmente libre). 


\section{Tabla 2 - Interpretación musical (continuación)}

\subsection{Ejecución musical}

3.2.1. Realización orientada en aspectos musicales

3.2.1.1. Orientación basada en las cualidades sonoras (Shakuhachi Zen ${ }^{6}$ )

3.2.1.2. Orientación en los existentes (música de época auténtica)

3.2.1.3. Orientación estructural (música contemporánea)

3.2.2. Realización orientada en aspectos funcionales

3.2.2.1. Funciones emotivas (canciones para funerales)

3.2.2.2. Funciones dinámicas (canciones de trabajo, de cuna)

3.2.2.3. Funciones semióticas (música para danza, teatro, cine, vídeo, multimedia)

3.2.3. Realización orientada en aspectos representativos

3.2.3.1. Orientación estética

3.2.3.2. Orientación referencial

3.2.3.3. Orientación educacional

3.3a. Inteligencia musical

3.3.1a. Estética musical

3.3.1.1a. Percepción estética

3.3.1.2a. Realización estética

3.3.1.3a. Teoría estética

3.3.2a. Pragmática musical

3.3.2.1a. Análisis

3.3.2.2a. Estudio de los problemas de ejecución

3.3.2.3a. Educación musical y crítica

3.3.3a Semiótica musical

3.3.3.1a. Significación musical intrínseca

3.3.3.2a. Referencia musical

3.3.3.3a. Interpretación musical

3.3b. Composición Musical

3.3.1b. Música absoluta

3.3.1.1b. Indeterminación total (Cage 4'33")

3.3.1.2b. Improvisación y formas abiertas

3.3.1.3b. Determinación completa de partitura

3.3.2b. Música funcional

3.3.2.1b. Música espontánea (función lúdica)

3.3.2.2b. Interacción música/función (música social, religiosa, militar, comercial)

6 Véase Gutzwiller (1984). 


\section{Tabla 2 - Interpretación musical (continuación)}

3.3.3b. Música representativa
$\begin{aligned} & \text { 3.3.3.1b. De la emoción (affektenlehre, ethos, rasa) } \\ & \text { 3.3.3.2b. Música descriptiva (poema sinfónico, música } \\ & \text { programática) }\end{aligned}$
$\begin{aligned} & \text { 3.3.3.3b. } \text { Sistemas simbólicos (raga, ópera de Beijin, } \\ & \text { gamelan, obra total wagneriana). }\end{aligned}$

\section{Referencias bibliográficas}

Boulez, Pierre (1986). Orientations, trad. M. Cooper, J.J. Nattiez (ed.). Cambridge: Harvard University Press.

Cage, John (1961). Silence. Middletown: Wesleyan University Press.

DougherTY, William P. (1993). «The Play of Interpretants: A Peircean Approach to Beethoven's Lieder». En The Peirce Seminar Papers: An Annual of Semiotic Analysis 1, M. Haley (ed.), 67-95. Oxford: Berg.

- (1994). «The quest for interpretants: Toward a Peircean paradigm for musical semiotics». Semiotica 99 (1/2), 163-184.

GuTZWILLER, A. (1984). «The shakuhachi of the Fuke-sect: Instrument of Zen». World of Music 26 (3).

Hanslick, Eduard (1989). Do Belo Musical, trad. N. Simone Neto. Campinas: UNICAMP.

HATtEN, Robert (1994). Musical Meaning in Beethoven: Markedness, Correlation and Interpretation. Bloomington: Indiana University Press.

Hicks, M. (1981). «Text, music, and meaning in the third movement of Luciano's Berio Sinfonia». Perspectives of New Music 20 (1/2), 199-224.

Kostelanetz, Richard (1988). Conversing with John Cage. New York: Limelight.

LIDOv, David (1986). «Music». En Encyclopedic Dictionary of Semiotics, T. Sebeok (ed.), 577-587. Amsterdam, Berlin, New York: Mouton de Gruyter. - (1987). «Mind and Body in Music». Semiotica 66 (1/3), 69-97.

Martínez, José Luiz (1991). Música \& Semiótica: Um Estudo Sobre A Questão da Representação na Linguagem Musical. Tesis de maestría no publicada, Pontifícia Universidade Católica de São Paulo.

- (1992). «Uma possível teoria semiótica da música (pautada logicamente em Charles Sanders Peirce)». Cadernos de Estudo, Análise Musical 5, 73-83 (São Paulo: Atravez).

- (1994). «Practicing Musical Semiotics». Musiikkitiede 6 (1/2), 158163. 
- (1996). «Icons in Music: a Peircean Rationale». Semiotica 110 (1/2), 57-86.

- (1997). Semiosis in Hindustani Music (= Acta Semiotica Fennica, 5). Imatra: International Semiotics Institute.

NATTIEZ, Jean-Jacques (1975). Fondements d'une sémiologie de la musique. Paris: Union Générale d'Éditions.

- (1990). Music and Discourse: Towards a Semiology of Music, trad. C. Abbate. Princeton: Princeton University Press.

PeIRCE, Charles S. (1938-1956). The Collected Papers, 8 vols., Charles Hartshorne, Paul Weiss, and Arthur W. Burks (eds.). Cambridge: Harvard University Press.

- (1982). Writings of Charles S. Peirce: A Chronological Edition, M.H. Fisch, E.C. More, C.J.W. Kloesel (eds.). Bloomington: Indiana University Press.

- (1992). The Essential Peirce, vol. 1, Nathan Houser and Christian Kloesel (eds.). Bloomington: Indiana University Press.

SCHNEIDER, Marius (1957). «Primitive Music». En New Oxford History of Music, vol. 1, E. Wellez (ed.), 1-82. London: Oxford Press.

TARASTI, Eero (1994). A Theory of Musical Semiotics. Bloomington: Indiana University Press. 